\title{
THE CAUCHY-RIEMANN EQUATIONS AND DIFFERENTIAL GEOMETRY
}

BY R. O. WELLS, JR.

1. Introduction. In 1907 Poincaré wrote a seminal paper [35] on various topics in several complex variables. In this paper we shall discuss Poincaré's paper and its influence and relationship to certain directions of research in complex analysis and geometry over the past 70 years. Poincarés paper discusses in some detail real 3-dimensional hypersurfaces in $\mathbf{C}^{2}$ and the relationship of the geometry of these hypersurfaces to the behavior of holomorphic functions and mappings defined near these hypersurfaces. The major topics treated by Poincaré include: (a) Hartogs' phenomenon, discovered only one year earlier by Hartogs [24]; (b) the Riemann mapping problem for domains in $\mathbf{C}^{2}$; (c) an analogous equivalence problem for smooth boundaries of such domains now known as the Poincare equivalence problem; (d) automorphisms or self-equivalences of domains and boundaries of domains (as in (c) and (d)); and (e) the tangential Cauchy-Riemann equations for a real hypersurface in $\mathbf{C}^{2}$.

The paper of Hartogs [24] and the paper of E. E. Levi [29] stimulated the major developments in several complex variables in this century. The general theory of several complex variables was summarized first by Osgood [36] who treated the early developments. In 1932 Behnke and Thullen wrote their famous monograph [4] which summarized the state of the art and asked the major questions which occupied researchers for the next 40 years. This culminated in the general theory of Stein manifolds in the 1950's (described, for instance, in Gunning and Rossi [23], Grauert and Remmert [21]) and the later significant interaction of several complex variables with modern partial differential equations (as described by Hörmander [26]).

Poincaré's work on Hartogs' theorem relates to the general theory of several complex variables mentioned above. His question about the equivalence of domains was taken up by Bergman and Carathéodory who developed their invariant metrics, both very useful in modern developments (see [3 and 11]). His question about the equivalence of boundaries stimulated significant work by Segre and Cartan in 1931-32 which we discuss in more detail later. On the whole, however, the problems proposed by Poincaré have remained dormant for some time while the general theory of several complex variables was being developed. In some sense he was asking more difficult questions whose solutions required invariants of a considerably higher order

Presented to the Symposium on the Mathematical Heritage of Henri Poincaré, April 7-10, 1980; received by the editors November 1, 1980.

1980 Mathematics Subject Classification. Primary 32-02, 53-01.

() 1982 American Mathematical Society $0273-0979 / 81 / 0000-0804 / \$ 04.25$ 
for their resolutions. In the past fifteen years these questions have been taken up again by a new generation of mathematicians who have a new perspective on these problems due to simultaneous developments in other areas of mathematics, such as differential topology, partial differential equations, algebraic topology, etc.

The study of the tangential Cauchy-Riemann equations on a real hypersurface, for instance, is a more recent phenomenon. Manifolds equipped with tangential Cauchy-Riemann equations and their abstractions are called CRmanifolds. Poincaré discussed the tangential Cauchy-Riemann equations in his paper, as is mentioned in $\$ 2$. These equations were not studied much at all until H. Lewy published his examples in 1956-60 [30, 31] which then stimulated a great deal of work. There were studies of the relation between function theory in the ambient space and the solutions of the tangential Cauchy-Riemann equations as well as investigations of the intrinsic questions concerning these equations (see the surveys by Folland and Kohn [20] and Wells [46] for further literature on this subject). Lewy's examples were also seminal for major developments in partial differential equations (see Hörmander [25]).

The question of the geometric equivalence of CR-manifolds (analogous to the equivalence of complex or Riemannian manifolds) is a question first raised by Poincaré in an extrinsic form in 1907. In this paper we want to give a summary of developments concerning this question. More generally we discuss the geometry of real hypersurfaces (considered as CR-manifolds), leaving the discussion of analysis on such manifolds to the works cited earlier.

In \$2 we give a summary of Poincaré's original 1907 paper, including the topics discussed above. In $\$ 3$ we discuss the solutions to the local equivalence problem for real hypersurfaces in $\mathbf{C}^{2}$ given by Segre and E. Cartan in 1931 and 1932 respectively. In $\$ 4$ we discuss the developments of the past two decades, when these questions were again taken up by a new generation of mathematicians.

2. Poincaré's paper of 1907. We want to formulate Poincaré's equivalence problem in $\mathbf{C}^{n}$, even though Poincaré and other researchers of the early 20th century confined their research almost exclusively to $\mathbf{C}^{2}$. Following Poincaré [35] we have

A. The local equivalence problem. Let $M$ and $M^{\prime}$ be two real-analytic real hypersurfaces defined in open sets $U$ and $U^{\prime}$ in $\mathbf{C}^{n}$. Given $p \in M$ and $p^{\prime} \in M^{\prime}$, when does there exist a neighborhood $V$ of $p$ and $V^{\prime}$ of $p^{\prime}$ and a biholomorphic mapping $F: V \rightarrow V^{\prime}$ so that $F(V \cap M)=V^{\prime} \cap M^{\prime}$ ? If such an $F$ exists then $M$ and $M^{\prime}$ are said to be locally equivalent at $p$ and $p^{\prime}$, respectively. If such an $F$ exists, then how unique is it?

B. The global equivalence problem. Suppose $M$ and $M^{\prime}$ are two real-analytic compact hypersurfaces in $\mathbf{C}^{n}$ and $M=\partial D, M^{\prime}=\partial D^{\prime}$ where $D$ and $D^{\prime}$ are bounded domains. When does there exist a neighborhood $V$ of $\bar{D}$ and $V^{\prime}$ of $\bar{D}^{\prime}$ and a biholomorphic mapping $F: V \rightarrow V^{\prime}$ such that $F(D)=D^{\prime}$ i.e., $\left.F\right|_{D}$ is a biholomorphic mapping onto $D^{\prime}$ ? 
C. The mixed equivalence problem. Suppose $M$ and $M^{\prime}$ are as in (B) above and suppose that for each point $p \in M, M$ is locally equivalent at $p$ to $M^{\prime}$ at some point $p^{\prime} \in M^{\prime}$. Then when is $M$ globally equivalent to $M^{\prime}$ in the sense of $(\mathrm{B})$ ?

If $n=1$, we see easily that two real-analytic arcs are always locally equivalent, since they are both locally equivalent to a segment of the real axis, by the very definition of real-analytic arc. The global equivalence problem is the Riemann mapping problem in the case $n=1$, and the mixed problem clearly doesn't have a solution unless there are global geometric hypotheses on $M$ and $M^{\prime}$ (e.g., for $n=1$ ). The obstruction to uniqueness of the local and global equivalence problem is the group (or pseudogroup) of local and global self-equivalences, which in the case of $n=1$, can be computed as a specific group of Möbius transformations (in the global case).

If $n>1$, Poincare observed that two local hypersurfaces are generically inequivalent, in contrast to the situation above. He based his observation on the fact that if $M$ and $M^{\prime} \subset C^{2}$ were locally equivalent, then the three local parameters describing $M^{\prime}$ would have to satisfy 4 real partial differential equations with respect to the 3 local parameters describing $M$. These are nothing but the tangential Cauchy-Riemann equations, expressed in terms of local coordinates on $M$ and $M^{\prime}$. Such an overdetermined inhomogeneous system does not generically have a solution, since the compatibility conditions are generically not satisfied. To this author's knowledge this is the first time the tangential Cauchy-Riemann equations appear in the literature, if only in a peripheral manner. As mentioned in the introduction one can consult [ 20 and 46] for surveys of the analysis of these equations.

Poincare discusses the solution of the equivalence problems above, and points out the need for a study of the automorphism groups of the structures involved. In particular, he singles out the distinction between an automorphism group or pseudogroup depending on a finite or an infinite number of parameters. If $M$ is a real hypersurface, then let $\operatorname{Aut}_{p}(M)$ be the pseudogroup of local self-equivalences or automorphisms at the point $p$. Then it's obvious that $M$ and $M^{\prime}$ being locally equivalent at $p$ and $p^{\prime}$ respectively implies that $\operatorname{Aut}_{p}(M) \cong \operatorname{Aut}_{p^{\prime}}\left(M^{\prime}\right)$. In other words the (pseudo-)group $\operatorname{Aut}_{p}(M)$ is an invariant of the structure and it can be used to derive other invariants. Let's consider the following two examples.

EXAMPLE 2.1. Let $z_{j}=x_{j}+i y_{j}, j=1,2$, be coordinates in $\mathbf{C}^{2}$, and let

$$
M=\left\{z \in \mathbf{C}^{2}: y_{2}=0\right\}
$$

be a real hyperplane in $\mathbf{C}^{2}$. Consider the local mapping

$$
T:\left\{\begin{array}{l}
\zeta_{1}=f\left(z_{1}, z_{2}\right) \\
\zeta_{2}=\phi\left(z_{2}\right)
\end{array}\right.
$$

where $f$ is holomorphic near $0 \in \mathbf{C}^{2}$, and $\phi$ is real-valued and real-analytic. Assume that $T$ is locally invertible at 0 . Then $T$ is the restriction to $M$ of a 
local biholomorphic mapping $\tilde{T}$ which maps $M$ to $M$ near $z=0$. For instance, a special case includes $T$ of the form

$$
T:\left\{\begin{array}{l}
\zeta_{1}=z_{1}+g\left(z_{2}\right) \\
\zeta_{2}=x_{2}
\end{array}\right.
$$

where $g$ is an arbitrary holomorphic function of $z_{2}$ near $z_{2}=0$. There are an infinite number of independent parameters in the coefficients of the Taylor expansion of $g$, and thus $\operatorname{dim} \operatorname{Aut}_{0}(M)=\infty$, in this case.

EXAMPLE 2.2. Let

$$
M=\left\{z \in \mathbf{C}^{2}: y_{2}=\left|z_{1}\right|^{2}\right\}
$$

be a parabolic hypersurface in $\mathbf{C}^{2}$. Poincare computed explicitly that $\operatorname{Aut}_{p}(M)$ $=H$ where $H$ is the group of transformations of $\mathbf{C}^{2}$ of the form

$$
\begin{aligned}
& \zeta_{1}=\frac{\lambda\left(z_{1}+a z_{2}\right)}{1-2 i \bar{a} z_{1}-\left(r+i|a|^{2}\right) z_{2}}, \\
& \zeta_{2}=\frac{|\lambda|^{2} z_{2}}{1-2 i \bar{a} z_{1}-\left(r+i|a|^{2}\right) z_{2}}
\end{aligned}
$$

$\lambda \in \mathbf{C}^{*}, a \in \mathbf{C}, r \in \mathbf{R}$. This is a Lie group, which is, in fact a subgroup of $S U(2,1)$ consisting of matrices of the form

$$
\left[\begin{array}{ccc}
\lambda & 0 & 0 \\
a & 1 & 0 \\
r+i|a|^{2} & -2 i \bar{a} & \bar{\lambda}^{-1}
\end{array}\right]
$$

which induce mappings of $\mathbf{C}^{2} \rightarrow \mathbf{C}^{2}$ which map $M$ to $M$ in terms of homogeneous coordinates considering $\mathbf{C}^{2} \subset \mathbf{P}_{2}$ in a natural manner, and $y_{2}=\left|z_{1}\right|^{2}$ being the restriction to $\mathbf{C}^{2}$ of a hyperquadric in $\mathbf{P}_{2}$. One main point of the computation of Poincaré is that $a$ priori the self-equivalences at 0 were only locally defined, whereas, in fact, all such are globally defined rational mappings which are nonsingular on $M$.

From the group-theoretic point of view one sees that Examples 2.1 and 2.2 are not locally equivalent at $0 \in \mathbf{C}^{2}$. This follows more simply from the Levi form, first discovered 2 years later. The Levi form is the first fundamental invariant of a real hypersurface, although this was not singled out explicitly by Poincaré in his paper.

The Levi form is defined as follows: if $M$ is a local hypersurface in $\mathbf{C}^{n}$ defined by

$$
M=\{z \in U: F(z)=0\}
$$

and $d F \neq 0$ on $M$, where $F$ is a real-valued $C^{2}$ function, then

$$
L(M)(t)=\sum_{i, j=1}^{n} \frac{\partial^{2} F}{\partial z_{i} \partial \bar{z}_{j}} t_{i} \bar{t}_{j}, \quad t \in \mathbf{C}^{n},
$$

where

$$
\sum_{i=1}^{n} \frac{\partial F}{\partial z_{i}} t_{i}=0
$$


This is an Hermitian form restricted to the holomorphic tangent space of $M$ (defined by (2.3)). This was defined for $n=2$ by Levi [29] and for $n>2$ by Krzoska [28]. The hypersurface $M$ is said to be strongly pseudoconvex if $L(M)$ is positive definite. The signature of $L(M)$ is a local biholomorphic invariant and an invariant of the local equivalence problem. In particular in Example 2.1 the Levi form has a zero eigenvalue, and in Example 2.2 the Levi form has a nonzero eigenvalue, so the signatures differ. The invariance of the signature is an easy fact to prove, and it is of critical importance in the geometric theory of functions of several complex variables.

In fact, Poincare showed that on a real hypersurface $M \subset \mathbf{C}^{2}$ there is a natural exterior differential system on $M$ (generated by the tangential holomorphic cotangent vectors and their complex conjugates) such that the complete integrability of this system was equivalent to the hypersurface being equivalent to Example 2.1. The obstruction to complete integrability of this exterior differential system is precisely the Levi form. This theorem was reformulated by Segre [36] and Cartan [12] sometime later in the form that a hypersurface in $\mathbf{C}^{2}$ is locally equivalent to a hyperplane (Example 2.1) if and only if the Levi form vanishes. This is also true in $\mathbf{C}^{n}$ (Sommer [38]).

Poincaré initiated a study of invariants of a real hypersurface by looking at relations between the Taylor series coefficients of a defining function $y_{2}=$ $F\left(z_{1}, x_{1}\right)$ and the Taylor series of a transformed equation $y_{2}^{\prime}=F^{\prime}\left(z_{1}^{\prime}, x_{1}^{\prime}\right)$. This he studied in terms of the coefficients of the expansion of the functions defining a local biholomorphic change of variables. By counting numbers of equations and unknowns he observed that the first nontrivial invariants (coefficients which couldn't be made to vanish by changes of coordinates) depended on the Taylor expansion up to order 9 in $\mathbf{C}^{2}$. This is not quite true, as the Levi form is of order 2, and the next higher order invariant in $\mathbf{C}^{2}$ turns out to be of order 6 (as was shown by E. Cartan in 1932). This process of finding invariants from the power series expansion point of view was carried out much later in a significant manner by Moser, as we see later in this paper. Basically, Poincaré recognized that at a point $p$ of a hypersurface $M$ there were an infinite number of nontrivial invariants $\left\{I_{j}\right\}$ which agrees with the corresponding invariants $\left\{I_{j}^{\prime}\right\}$ at any locally equivalent hypersurface $M^{\prime}$ at a specific point $p^{\prime}$. To determined what these invariants are and how to compute them has been the object of much of the research of the ensuing 70 years and will be discussed in the later sections of this paper.

In 1906, a year before Poincaré's paper, Hartogs gave the first examples of simultaneous analytic continuation of holomorphic functions of more than one variable, the study of which dominated research in several complex variables for the next 50 years as discussed earlier. The general Hartogs' theorem asserts that if $D$ is a bounded domain in $\mathbf{C}^{n}$ with smooth boundary, such that $\mathbf{C}^{n}-D$ is connected, then any function $f$ holomorphic near $\partial D$ continues analytically in a single-valued manner to a holomorphic function on $D$. Poincaré gave a brief proof of the general result, and a much longer proof of the same result for the unit ball in $\mathbf{C}^{n}$ using spherical harmonics. The reason for the second proof is that there was no accepted rigorous proof of the general statement until Brown [7] and Bochner [5] gave their proofs thirty years later (it's now “elementary", cf. Hörmander [26]). 
In the remainder of this wide-ranging paper Poincare discussed among other things, the problem of when a perturbation of the sphere is still globally equivalent to the sphere. He sets up some nonlinear equations, and discusses, using spherical harmonics, a characterization of the linearization of the relevant equations, i.e., a first order solution to this particular equivalence problem in this special geometric setting. This was a first order approximation to a class of problems which have been resolved to any extent only quite recently (Webster [43], Burns, Shnider and Wells [10], Johnson [27], Greene and Krantz [22]) as is discussed in the next section.

3. Solutions of the Poincaré equivalence problem by B. Segre and E. Cartan. We now want to give a brief overview of two different types of solutions to the equivalence problem of Poincaré as formulated in the previous section. Let's restrict our attention to real-analytic hypersurfaces in $\mathbf{C}^{\mathbf{n}}$. Many of the concepts and results are valid for more general smooth manifolds, but there are sometimes subtleties which we won't concern ourselves with here.

Let $M$ be a (real-analytic) hypersurface in some open set in $\mathbf{C}^{n}$, i.e. for $\boldsymbol{F}$ a real-valued function in an open set $D \subset \mathbf{C}^{n}$,

$$
M=\{z \in D: F(z)=0, d F \neq 0\} .
$$

Consider the bundle $H(M) \subset T(M)$ defined by $H(M)=T(M) \cap J T(M)$ where $J: T(M) \rightarrow T(M)$ is the real-linear mapping corresponding to the multiplication of vectors by $i$ (i.e., $J^{2}=-I$ ). Thus

$$
\left.H(M) \subset T(M) \subset T\left(\mathbf{C}^{n}\right)\right|_{M}
$$

and $H(M)_{x}$ is the maximal complex subspace of $T\left(C^{n}\right)_{x}$ which is contained in $T(M)_{x}$ for $x \in M$. The bundle $H(M)$ is called the holomorphic tangent bundle to $M$. The pair $H(M) \subset T(M)$ is called the CR-structure (Cauchy-Riemann structure) of the hypersurface $M$ (the name arises from the fact that sections of $\overline{H(M)}$ in the natural conjugations of vector fields on $\mathbf{C}^{n}$ are the tangential Cauchy-Riemann equations, alluded to above). We call $M$ with its CR-structure a $C R$-hypersurface. The notion of CR-hypersurface can be abstracted in an appropriate manner, just as Riemannian manifolds are abstractions of submanifolds with the induced Riemannian metric. An abstract CR-hypersurface is a real $(2 n-1)$-manifold with a subbundle $H(M) \subset T(M)$ where $H(M)$ is a complex vector bundle of complex rank $(n-1)$. The CR-structure is integrable if $[\Gamma(H(M)), \Gamma(H(M))] \subset \Gamma(H(M))$. This is automatic for hypersurfaces in $\mathbf{C}^{n}$ as is easily checked. An integrable abstract $C R$-hypersurface is the correct abstraction of a hypersurface with respect to the class of problems raised by Poincaré. For simplicity we will speak simply of "CRhypersurfaces".

Just as with the isometric embedding problem one wants to know if all of the abstract ones can be embedded. This is a subject of current interest and is not completely resolved (see [6, 2] for some recent results). We mention here that for $\operatorname{dim} M=3$ the answer is no as is seen by an example of L. Nirenberg [33].

If $M, M^{\prime}$ are two CR-hypersurfaces and $f: M \rightarrow M^{\prime}$ is a smooth mapping, then $f$ is a CR-mapping if $\left.d f\right|_{H(M)}$ is a complex-linear mapping from $H(M) \rightarrow$ $H\left(M^{\prime}\right)$. This is a natural generalization of the notion of holomorphic mapping 
$g$ of $\mathbf{C}^{n}$ to $\mathbf{C}^{n}$ where $d g$ is required to be $\mathbf{C}$-linear at each point of $\mathbf{C}^{n}$ for $\boldsymbol{g}$ to be holomorphic. A CR-equivalence is simply a CR-mapping $f: M \rightarrow M^{\prime}$ such that $f^{-1}$ exists and is also a CR-mapping.

The first fundamental result in the local Poincare equivalence problem as posed in $\$ 2$ is that $M$ and $M^{\prime}$ are equivalent in the sense of Poincare if and only if they are CR-equivalent. This latter is an intrinsic notion, and this result was proved by Segre in 1931 [37] in $C^{2}$ and by Tanaka [39] for $n>2$. It is an application of the Cauchy-Kowaleski theorem, or the equivalent CartanKähler theorem. Thus the extrinsic equivalence of Poincaré can be reduced to an intrinsic study of the geometry of the manifold itself. The invariants which are described below are all invariants of the intrinsic CR-structure, although some of them can be expressed in terms of extrinsic structure (like Moser's normal coordinates). Segre, in the same paper, showed that if the Levi form is nondegenerate then the local automorphism group of CR-mappings $\operatorname{Aut}(M)$ is finite dimensional, and if the Levi form is identically zero then $M$ is equivalent to the hyperplane with its infinite-dimensional automorphism group, as was mentioned earlier. The finite-dimensional result used a deep finiteness criterion for a transformation group leaving invariant solutions to certain second order ordinary differential equations due to Tresse [42]. An example of this is the differential equation $y^{\prime \prime}=0$, whose solutions have graphs which are straight lines in the plane, and the groups of diffeomorphism of the plane which leave the straight lines invariant is the projective group which is a finite-dimensional Lie group. Segre also constructed for each hypersurface $M \subset \mathbf{C}^{2}$, a family of analytic varieties in $\mathbf{C}^{2}$ parametrized by the points of $M$ and such that CR-equivalence of the hypersurface was characterized by the biholomorphic equivalence of these varieties. These "algebro-geometric invariants" were utilized later in Webster's solution of the global equivalence problem for ellipsoids [43].

One year later E. Cartan gave a complete solution to the equivalence problem in terms of differential-geometric invariants. He constructed a suitable bundle of frames $B$ adapted to the CR-structure of $M \subset C^{2}$ and a natural (Cartan) connection $\omega$ and curvature $\Omega$ on this bundle $B \rightarrow M$ such that $M$ and $M^{\prime}$ are CR-equivalent if and only if there is a bundle equivalence $B \stackrel{\approx}{\rightarrow} B^{\prime}$ which preserves the connection (and hence the curvature). This is completely analogous to the construction of a connection and curvature on the orthonormal frame bundle of a Riemannian manifold, where the curvature and its covariant derivatives are a complete set of invariants for Riemannian geometry. The major difference is that the bundle $B$ for the CR-geometry is a 2nd order frame bundle, i.e., is a bundle of frames for the total space of a line bundle $L$ determined by the (first order) CR-structure, namely $L$ is the 1 real-dimensional subbundle of the cotangent bundle annihilated by the holomorphic tangent vectors. This was carried out in lengthy detail in two long papers [12] with many applications and examples worked out. He obtained the finiteness results mentioned earlier independent of the lengthy Tresse monograph. In these papers is also a complete classification of the hypersurfaces for which $\operatorname{Aut}(M)$ is transitive, which can be reduced to solvable algebraic problems. Moreover, the curvatures are defined on the frame bundle, but for a generic class of hypersurfaces (called nonumbilic, where the 
lowest order curvature is not zero) one can construct 9 scalar invariants, analogous to Gaussian curvature (or more generally scalar curvature) for Riemannian surfaces. These are functions defined on $M$ so that $M$ and $M^{\prime}$ are equivalent if and only if the scalar invariants are pointwise preserved. These scalar invariants are of 7 th and 8 th order, i.e., depend on 7 th and 8 th order derivatives of the defining function. On the other hand if the lowest order curvature terms vanish identically, then Cartan showed that the surface was locally equivalent to the "local model", the hyperquadric. In fact, the curvatures measure the deviation from the local model. The nondegeneracy of the Levi form insured that there was a good approximating hyperquadric, and the curvatures measure the deviation, just as in Riemannian geometry the curvature measures the deviation from a first or ler approximation by a plane. Cartan worked entirely intrinsically (often using extrinsic quantities to represent the intrinsic differential forms, however) and did not concern himself too much with the nature of the embedding of $M$ in $\mathbf{C}^{2}$, except in his description of examples.

4. Higher-dimensional results in the past two decades. More recently there has been a lot of activity by various mathematicians extending the Segre and Cartan solutions to the Poincaré equivalence problems for hypersurfaces in $\mathbf{C}^{2}$ to hypersurfaces in $\mathbf{C}^{n}$ as well as applications of these results. In 1962 Tanaka [39] generalized Cartan's differential-geometric invariants to a special class of hypersurfaces in $\mathbf{C}^{n}$ (equipped with a 1-parameter family of automorphisms of the structure). In 1965 Tanaka announced the solution to the general local equivalence problem in $\mathbf{C}^{n}$ [40]. The details appeared in a long paper in 1976 in a very general setting of which the CR-hypersurface equivalence problem was a special case [41]. In the meantime Chern and Moser in 1974 [14] presented a dual version of the differential-geometric solution to the equivalence problem which included two main constructions: (a) the principal bundle with Cartan connection and curvatures on an appropriate frame bundle just as in Cartan for $\mathbf{C}^{2}$ and equivalent to Tanaka's work mentioned above (Tanaka's work is less explicit for real hypersurfaces), (b) a normal form called the Moser normal form (cf. [32]) which is a normalization of the Taylor series of the defining function for a real hypersurface with nondegenerate Levi form near a point $p_{0}$ up to the action of the finite-dimensional Lie group acting on $\mathbf{C}^{n}$ which leaves the best approximating hyperquadric to $M$ at $p_{0}$ fixed. For instance in $\mathbf{C}^{2}$, Moser's normal form looks like the hypersurface given by

$$
v=F(z, u)=z \bar{z}+C_{42} z^{4} \bar{z}^{2}+C_{24} z^{2} \bar{z}^{4}+\sum_{k+l>7} C_{k l} z^{k} \bar{z}^{l}
$$

where $z=x+i y, w=u+i v$ are coordinates for $\mathbf{C}^{2}$, and $F$ is a real-valued function of $(z, u)$, and the coefficients $C_{k l}$ are functions of $u$. The coefficients are unique up to the action of the group acting on $\mathbf{C}^{2}$ given in (2.1). The coefficients $\left\{C_{k l}\right\}$ are "curvatures". If they vanish then the hypersurface is equivalent to a hyperquadric (patently obvious in the expansion above). These normal form coefficients are equivalent to the curvatures and higher 
covariant derivatives of the curvature being evaluated for a specific choice of frame compatible with the CR-structure (just as for normal coordinates in Riemannian geometry).

In 1975 Chern [13] generalized the Segre invariants to $\mathbf{C}^{n}$ and studied these invariants in the language of exterior differential systems. Cartan had remarked in the introduction to his paper [12] that Segre's invariants were not a complete set of invariants (while Segre had claimed they were!). In fact this point was clarified only recently by Faran [16] who showed that, for instance in $\mathbf{C}^{2}$, there is an example of two distinct $\mathrm{CR}$-structures on a specific 3-dimensional manifold (which the Cartan invariants distinguish), but for which the Segre invariants coincide. Moreover, this is the worst discrepancy possible for the two systems of invariants. Similarly, in higher dimensions the distinction between the invariants is a discrete phenomenon. Thus local variation in CR-structures can be described by either type of invariants.

We now turn to applications of these local invariants to various versions of the global equivalence problem. First, Webster used the Segre type invariants to classify the set of all real ellipsoids in $\mathbf{C}^{n}$ with respect to $\mathrm{CR}$-equivalence [43]. Burns and Shnider [9] gave a general classification of real hypersurfaces $M$ in $\mathbf{C}^{n}$ where $\operatorname{Aut}(\boldsymbol{M})$ is transitive, generalizing Cartan's classification in $\mathbf{C}^{2}$. The scalar invariants of Cartan were generalized to $\mathbf{C}^{n}$ for nonumbilic hypersurfaces (the lowest order curvature invariants were not zero in an appropriate sense; see Burns, Shnider and Wells [10] and Webster [45]). In particular this involved a refinement of Moser's normal form. By using Thom transversality and the scalar invariants one finds that "almost all" deformations of a given CR-hypersurface are inequivalent CR-hypersurfaces and whose automorphism group consists only of the identity transformation [10]. Fefferman proved an important extension theorem [17] for strongly pseudoconvex domains with smooth boundary. Namely a biholomorphic equivalence of such domains extends to a CR-equivalence of their boundaries. Thus the invariants on the boundaries in such an equivalence must be preserved. This gives a way of distinguishing different complex structures on the interior of such domains. In Burns, Shnider and Wells [10] it was shown by using the scalar invariants, Thom transversality, and Fefferman's extension theory that there is an infinite-dimensional family of perturbations of the boundary of the open unit ball in $\mathbf{C}^{n}$ (or any strongly pseudoconvex domain) so that the deformed domains are biholomorphically inequivalent and have no automorphisms. Johnson wrote down explicit algebraic examples of such deformations in [27], basing his construction on earlier results of Webster who studied invariants in specific geometric settings [45]. For instance, one finds in [27], if

$$
p(z)=z_{1}^{5}+z_{1}^{3}+z_{1}^{2}+z_{1} z_{2}+z_{2} z_{3}+z_{3} z_{4}
$$

\footnotetext{
${ }^{1}$ This result was recently improved significantly by $S$. Bell, who showed that a biholomorphic mapping from a strongly pseudoconvex domain to a domain with a smooth boundary extends to the boundary. In particular the image domain must be strongly pseudoconvex, which was not hypothesized.
} 
is a polynomial in $\mathbf{C}^{4}$, and if we set

$$
D_{t}=\left\{z \in \mathbf{C}^{4}:|z|^{2}+t^{2}|p(z)|^{2}<1\right\}
$$

for $0 \leqslant|t|<\varepsilon$, for $\varepsilon$ sufficiently small, then $D_{t}$ is a 1 -parameter family of strongly pseudoconvex domains in $\mathbf{C}^{4}$ with $D_{t}$ not biholomorphic to $D_{t^{\prime}}$ for $t \neq t^{\prime}$, and with $\operatorname{Aut}\left(D_{t}\right)=\{\mathrm{id}\}, t \neq 0$. However, as is clear from the definition, $D_{0}$ is the unit ball in $C^{4}$ with $\operatorname{Aut}\left(D_{0}\right)=S U(4,1) /($ finite subgroup) of real dimension 24. All of the $D_{t}$ have real-analytic boundaries and are diffeomorphic to the unit ball. This sheds some light on the subtlety of the original global Poincaré equivalence problem. Recently, Greene and Krantz [22] showed that the set of strongly pseudoconvex domains with no automorphisms is open in the set of such domains in general, while the set of domains equivalent to a given domain is a closed set. This complements the result in [10] that domains with no automorphisms are dense, as discussed above.

In the study of CR-structures on hypersurfaces the Cartan type invariants are described in terms of a principal bundle, a connection, and a curvature, just as in Riemannian geometry. In the CR-geometry there is a distinguished family of real curves, called chains, which are analogous to geodesics in a Riemannian manifold. The chains are invariants of the CR-structure and defined by second order differential equations depending on the connection in an appropriate manner (Cartan [12], Chern and Moser [14]). For the hyperquadric these chains are simply the intersection of the real hypersurface with complex lines in the ambient space, although this is not true in general. Fefferman [18] showed that if $M$ is a strongly pseudoconvex manifold, then there is a naturally defined Lorentz metric on $M \times S^{1}$ such that the projection of the light rays (for the Lorentz metric) on $M \times S^{1}$ to $M$ are precisely the chains of the CR-structure of $M$.

In a different direction, and related to the extension theorem mentioned earlier, Fefferman [17] found an asymptotic expansion of the Bergman kernel of a strongly pseudoconvex domain $D$ in terms of the distance to the boundary $\rho=d(z, \partial D)$. This involved powers of $\rho$ and also a logarithmic term. In [19] Fefferman undertook a deep and not yet well understood study of the relations between the coefficients of the expansion of the Bergman kernel and the invariants of the CR-structure on the boundary. This is a complex story, and the ramification and meaning of the relationships developed has yet to be explored.

Two additional topics are worth mentioning which relate to the variation in CR-structures. First, Dippolito [15] has taken an initial step in a description of the (infinite-dimensional) moduli space for generic classes of CR-structures on a CR-hypersurface. He obtains "coordinates" for the moduli structure by looking at transversal sections to orbits under the appropriate Lie group of the Moser normal coordinates in a suitable jet bundle. In a second direction there is a relationship of variation of CR-structure to descriptions of real spacetimes in relativity theory. Namely Penrose observed that if $H$ is a spacelike hypersurface in a Lorentzian 4-dimensional real vacuum spacetime $S$ (i.e., the Ricci curvature of the Lorentzian metric $d s^{2}$ is zero), then one can associate to each point of $S$ the 2-dimensional cone of light rays emanating 
from that point (the cones of tangent vectors annihilated by $d s^{2}$ ). The totality of light rays for variable points of $H$ can be given the structure of a 5-dimensional real manifold equipped with an integrable CR-structure with nondegenerate Levi form. Thus, to each $H$ there is an $M_{H}$ with a full set of invariants of either Segre or Cartan type. As $H$ varies in $S$ (is evolved by Einstein's equations), the $M_{H}$ varies in the space of moduli of such structures (parametrized suitably by its CR-invariants). This has been described by LeBrun [28a] explicitly and by Bryant [8], who describes this in Cartan's language of moving frames. This interplay between real spacetime and CR-structures is a refinement of an earlier relationship between variations in complex structure on a 3-dimensional open complex manifold in $\mathbf{P}_{\mathbf{3}}(\mathbf{C})$ and holomorphic solutions of Einstein's self-dual vacuum field equations (Penrose [34]; see Atiyah [1], Wells [47, 48] for expository descriptions of this recent relationship between complex geometry and mathematical physics).

Perhaps this relation between CR-structures and spacetimes will have some positive effects on our understanding of relativity theory and the difficult question of the relation between gravity and quantum mechanics (cf. Penrose [34]). This would be a pretty state of affairs to have Poincaré having asked questions in an area of pure mathematics which in the long run had applications to the description of the universe we live in as he was himself very involved in such problems throughout his career as a mathematician, physicist, and philosopher.

\section{REFERENCES}

1. M. F. Atiyah, Geometry of Yang-Mills fields, Lezioni Fermione, Acad. Naz. dei Lincei, Scuola Normale Sup., Pisa, 1979.

2. M. S. Baouendi and F. Treves, A property of the functions and distributions annihilated by a locally integrable system of complex vector fields, Ann. of Math. (2) 113 (1981), 387-421.

3. S. Bergman, The kernel function and conformal mapping, Math. Surveys, no. 5, Amer. Math. Soc., Providence, R.I., 1950.

4. H. Behnke and P. Thullen, Theorie der Funktionen mehrerer komplexer Veränderlichen, 2nd ed., Ergebnisse der Math. und ihrer Grenzgebiete Band 51, Springer-Verlag, Berlin-New York, 1970.

5. S. Bochner, Analytic and meromorphic continuation by means of Green's formula, Ann. of Math. (2) 44 (1943), 652-673.

6. L. Boutet de Monvel, Integration des equations de Cauchy-Riemann induites formelles, Sèminaire Goulaouic-Lions-Schwartz (1974-75), Exposé No. 9, Centre Math. Ecole Polytech., Paris, 1975.

7. A. Brown, On certain analytic continuations and analytic homeomorphisms, Duke Math. J. 2 (1936), 20-28.

8. R. Bryant, Space-times and CR-manifolds, Trans. Amer. Math. Soc. (to appear).

9. D. Burns and S. Shnider, Real hypersurfaces in complex manifolds, Proc. Sympos. Pure Math., vol. 30, Amer. Math. Soc., Providence, R. I., 1977, pp. 141-168.

10. D. Burns, S. Shnider and R. O. Wells, Jr., Deformations of strongly pseudoconvex domains, Invent. Math. 46 (1978), 237-253.

11. C. Carathéodory, Über das Schwarze Lemma bei analytischen Funktionen von zwei komplexen Veränderlichen, Math. Ann. 97 (1926), 76-98.

12. E. Cartan, Sur la géométrie pseudo-conforme des hypersurfaces de deux variables complexes. I, Ann. Math. Pura Appl. (4) 11 (1932), 17-90 (or Oeuvres. II, 2, 1231-1304); II, Ann. Scuola Norm. Sup. Pisa, (2) 1 (1932), 333-354 (or Oeuvres. III, 2, 1217-1238). 
13. S. S. Chern, On the projective structure of a real hypersurface in $\mathbf{C}_{n+1}$, Math. Scand. 36 (1975), 74-82.

14. S. S. Chern and J. Moser, Real hypersurfaces in complex manifolds, Acta Math. 133 (1974), 219-271.

15. P. Dippolito, Universal bundles for deformations of asymetric structures, Trans. Amer. Math. Soc. (to appear).

16. James Faran, Segre families and hypersurfaces, Invent. Math. 60 (1980), 135-172.

17. C. Fefferman, The Bergman kernel and biholomorphic mappings of pseudoconvex domains, Invent. Math. 26 (1974), 1-65.

18. __ Monge-Ampère equations, the Bergman kernel, and geometry of pseudoconvex domains, Ann. of Math. (2) 103 (1976), 395-416.

19. , Parabolic invariant theory in complex analysis, Advances in Math. 31 (1979), 131-262.

20. G. B. Folland and J. J. Kohn, The Neumann problem for the Cauchy-Riemann complex, Ann. of Math. Studies, no. 75, Princeton Univ. Press, Princeton, N. J., 1972.

21. H. Granert and R. Remmert, Analytische Stellenalgebren, Springer, Berlin-Heidelberg-New York, 1971.

22. R. Greene and S. Krantz, Deformation of complex structures, estimates for the $\bar{\partial}$-equation, and stability of the Bergman kernel, Adv. in Math. (to appear).

23. R. C. Gunning and Hugo Rossi, Analytic functions of several complex variables, PrenticeHall, Englewood Cliffs, N. J., 1965.

24. F. Hartogs, Zur Theorie der analytischen Funktionen mehrerer unabhängiger Veränderlichen insbesondere über die Darstellung derselben durch Reihen, welche nach Potenzen einer Veränderlichen fortschreiten, Math. Ann. 62 (1906), 1-80.

25. L. Hörmander, Linear partial differential operators, Springer-Verlag, Berlin-GöttingenHeidelberg, 1963.

26. __ An introduction to complex analysis in several variables, North-Holland, Amsterdam, 1973.

27. David S. Johnson, Biholomorphic equivalence in a class of graph domains, Indiana J. Math. 29 (1980), 341-348.

28. A. Krzoska, Uber die naturlichen Grenzen der analytischen Funktionen mehrerer Veränderlicher, Dissertation, Greifswald, 1933.

28a. C. R. LeBrun, Jr., Spaces of complex geodesics and related structures, Thesis, Oxford University, 1980.

29. E. E. Levi, Studii sui punti singolari essenziale delle funzioni analitiche di due o più variabili complesse, Annali di Mat. 17 (1909), 61-87.

30. H. Lewy, On the local character of the solutions of an atypical linear differential equation in three variables and a related theorem for regular functions of two complex variables, Ann. of Math. (2) 64 (1956), 514-522.

31. , On hulls of holomorphy, Comm. Pure. Appl. Math. 13 (1960), 587-591.

32. J. Moser, Holomorphic equivalence and normal forms of hypersurfaces, Proc. Sympos. Pure Math., vol. 27, Part 2, Amer. Math. Soc., Providence, R. I., 1975, pp. 109-112.

33. L. Nirenberg, Lectures on linear partial differential equations, CBMS Reg. Conf. Ser. in Math., no. 17, Amer. Math. Soc., Providence, R. I., 1973.

34. R. Penrose, Nonlinear gravitons and curved twistor theory, Gen. Relativity and Gravitation 7 (1976), 31-52.

35. H. Poincaré, Les functions analytique de deux variables et la représentation conforme, Rend.

Circ. Math. Palermo 23 (1907), 185-220 (or Oeuvres. IV, 244-289).

36. W. F. Osgood, Lehrbuch der Funktionentheorie, 2nd. ed., vol. 2, part I, Teubner, Leipzig, 1929.

37. B. Segre, Questioni geometriche legate colla teoria delle funzioni di due variabili complesse, Rend. Sem. Mat. Roma 7 (1931), 59-107.

Interno al problema di Poincaré della rappresentazione pseudoconforme, Rend. Acc. Lincei 13 (1931), pp. 676-683.

38. F. Sommer, Komplex analytische Blätterung reeler Mannigfaltigkeiten im $\mathbf{C}^{n}$, Math. Ann. 136 (1958), 111-133. 
39. N. Tanaka, On the pseudo-conformal geometry of hypersurfaces of the space of $n$ complex variables, J. Math. Soc. Japan 14 (1962), 397-429.

40. ㄴ, Graded Lie algebras and geometric structures, Proc. U.S.-Japan Seminar in Differential Geometry, (Kyoto, 1965), Nippon Hyoronsha, Tokyo, 1966, pp. 147-150.

41. On non-degenerate real hypersurfaces, graded Lie algebras, and Cartan connections, Japan. J. Math. 2 (1976), 131-190.

42. M. A. Tresse, Détermination des invariants ponctuels de l'equation différentielle ordinaire du second order $y^{\prime \prime}=w\left(x, y, y^{\prime}\right)$, S. Hirzel, Leipzig, 1896.

43. S. M. Webster, On the mapping problem for algebraic real hypersurfaces, Invent. Math. 43 (1977), 53-68.

44. __ On the Moser normal form at a non-umbilic point, Math. Ann. 233 (1978), 97-102.

45. _ The rigidity of CR-hypersurfaces in a sphere, Indiana Univ. Math. J. 28 (1979), 405-416.

46. R. O. Wells, Jr., Function theory on differentiable submanfolds, Contributions to Analysis, Academic Press, New York, 1974, pp. 407-441.

47. , Complex manifolds and mathematical physics, Bull. Amer. Math. Soc. (N. S.) 1 (1979), 296-336.

48. , Complex geometry in mathematical physics, Univ. of Montreal Press (to appear).

Department of Mathematics, Rice University, Houston, Texas 77001 
